
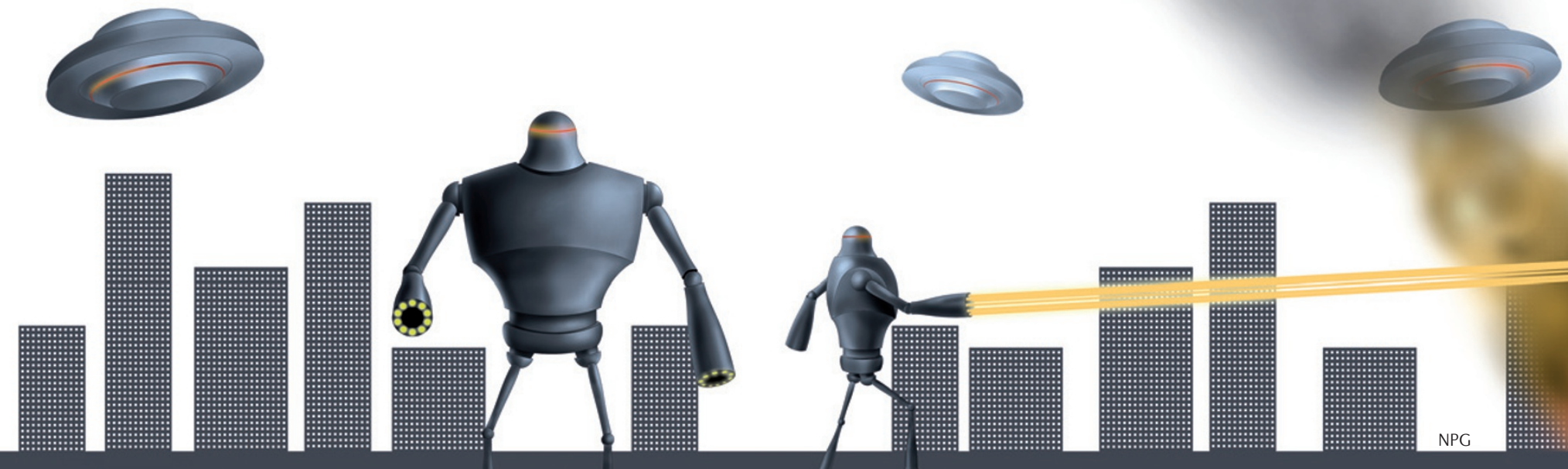

NPG

\title{
Resistance is futile
}

Secondary bacterial pneumonia is a common complication of influenza virus infection in humans. But what accounts for the increased morbidity that is associated with viral-bacterial co-infection? Now, Medzhitov and colleagues describe a mouse model of co-infection with influenza virus and Legionella pneumophila that can be used to discriminate between the ability of the host to detect and eliminate a pathogen (resistance) and the ability to maintain homeostasis with a given level of pathogen burden (tolerance).

All mice that had been infected intranasally with a sublethal dose of influenza virus followed 3 days later by a sublethal dose of L. pneumophila died within a week of co-infection, whereas all mice survived a single infection with the virus or the bacteria. Importantly, there was no significant difference in the viral or bacterial burden after single infection or co-infection, and co-infection was not associated with systemic dissemination of either pathogen. Furthermore, an attenuated strain of L. pneumophila that is unable to secrete virulence factors still resulted in $100 \%$ mortality in co-infected mice. So, bacterial growth or virulence is not required for the effects of co-infection, which confirms that this is not a failure of immune resistance.

Using various mouse strains with genetic deletions of key immune molecules, as well as antibodymediated depletion of neutrophils or natural killer cells, the authors showed that co-infection still results in mortality in the absence of all major immune and inflammatory pathways that might be triggered by the virus or bacteria. They suggest that these results show that an excessive inflammatory response leading to immunopathology is not the cause of death after co-infection. Even in the absence of all controllable immunostimulatory signals - when immunodeficient (Toll-like receptor 2 $(\text { Tlr } 2)^{-/-}$Tlr $4^{-/-}$) mice were infected with influenza virus and a severely

tolerance can determine the outcome of an infection independently of resistance and is, thus, a bona fide host defence strategy attenuated L. pneumophila strain that lacked flagellin and was unable to replicate or secrete effectors - most mice died following co-infection but not after a single infection with either the virus or the bacteria.

So, if resistance is not involved in co-infection-associated mortality in terms of the failure of the immune response to control pathogen growth and spread, increased pathogen virulence or the effects of immunopathology - the alternative scenario is that the host is unable to tolerate the tissue damage that results from co-infection. The lungs of co-infected mice had significantly increased necrosis of airway epithelial cells compared with singly infected mice, and genes involved in tissue protection and repair were downregulated in co-infected mice compared with singly infected mice. Administration of amphiregulin — an epidermal growth factor family member that has a role in maintaining lung homeostasis during influenza virus infection - to Tlr2 $2^{-/-} \mathrm{Tlr}^{-/-}$mice that had been infected with influenza virus and the severely attenuated L. pneumophila strain significantly decreased lung damage and mortality but had no effect on viral or bacterial burden.

Together, the results show that tolerance can determine the outcome of an infection independently of resistance and is, thus, a bona fide host defence strategy that could be targeted for therapeutic purposes.

\section{Kirsty Minton}

ORIGINAL RESEARCH PAPER Jamieson, A. M. et al. Role of tissue protection in lethal respiratory viralbacterial coinfection. Science 25 Apr 2013 (doi:10.1126/science.1233632)

FURTHER READING Schneider, D. S. \& Ayres, J. S. Two ways to survive infection: what resistance and tolerance can teach us about treating infectious diseases. Nature Rev. Immunol. 8, 889-895 (2008) 